\title{
New clues for flu vaccine design
}

\begin{abstract}
Multiple subtypes and strains of influenza exist and new strains are constantly emerging. The development of vaccines providing long-term broad protection against influenza remains a key therapeutic goal. Now, a trio of studies have identified human antibodies exhibiting broadly neutralizing activity, which may have implications for future vaccine design.

Type A influenza viruses are the most common and virulent. They are divided into two groups, which are classified into subtypes based on the surface proteins haemagglutinin (HA) and neuraminidase, and many different strains exist. The HA globular head mediates virus attachment to target cells through interactions
\end{abstract}

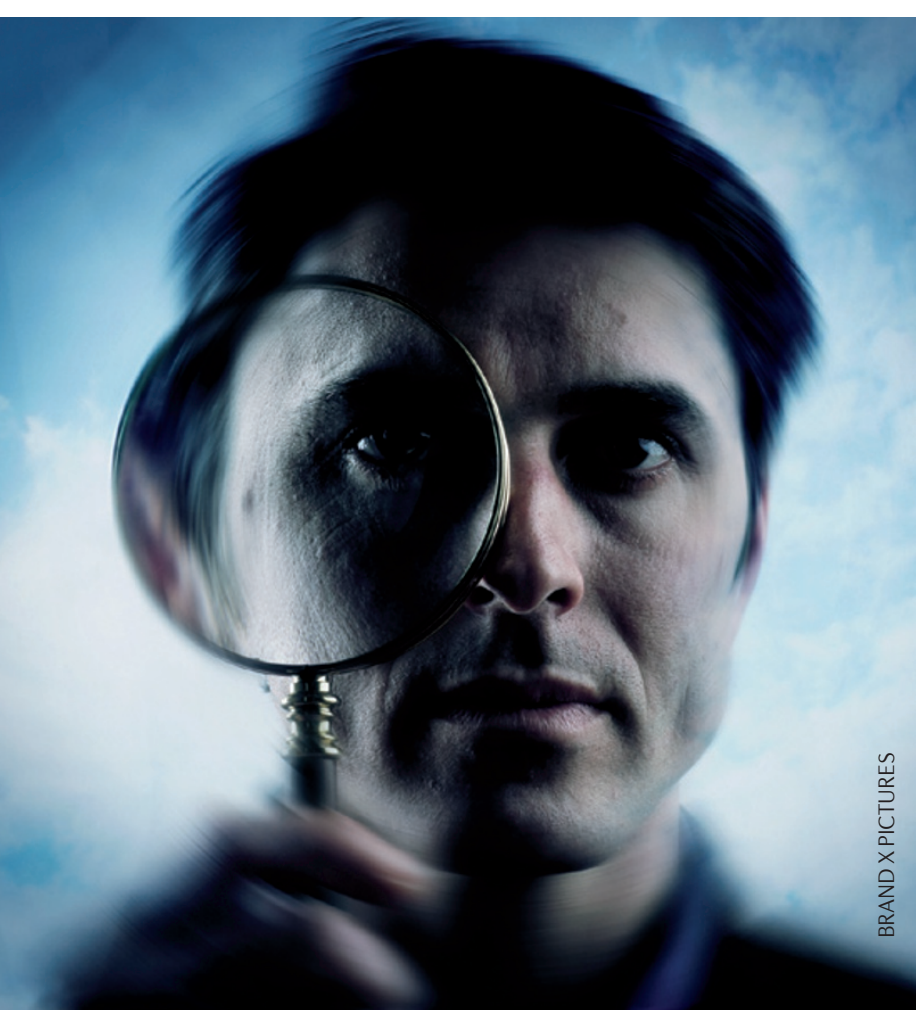

with sialic acid receptors. Most of the current neutralizing antibodies bind to exposed loops surrounding the receptor binding site of the HA globular head and interfere with attachment. However, these loops are highly variable and so such antibodies are typically strain-specific. New vaccines must therefore be generated annually, based on predictions of which virus strains are most likely to be in circulation. As a result, such vaccines provide little or no protection against unforeseen strains.

In the first paper, Ekiert et al. generated stable monoclonal antibody (mAb)-producing human memory $\mathrm{B}$ cells from the blood of donors who were recently vaccinated against influenza and isolated HA-reactive clones of the $\mathrm{H} 3$ serotype. They identified the mAb CR8020, which exhibited high-affinity binding to influenza A group 2 (but not group 1) subtypes, including $\mathrm{H} 3$ isolates spanning 50 years of virus evolution. CR8020 binds to a highly conserved epitope at the base of the HA stem, in close proximity to the viral membrane (lower down the stalk than any other characterized influenza antibody), inhibiting conformational changes in $\mathrm{HA}$ and blocking membrane fusion and viral entry. CR8020 protected mice against a high lethal dose of the $\mathrm{H} 3 \mathrm{~N} 2$ and H7N7 strains when it was administered pre- and post-infection.

Meanwhile, Corti et al. developed a novel high-throughput single-cell culture method to screen 104,000 plasma cells from eight donors who had previously produced a strong heterosubtypic antibody response, following natural influenza $\mathrm{A}$ infection or vaccination. They isolated an extremely rare mAb - FI6 - which recognized all $16 \mathrm{HA}$ subtypes of influenza A, neutralizing with an unprecedented breadth all of the group 1 and group 2 viruses tested. Co-crystallization with $\mathrm{H} 1$ and $\mathrm{H} 3$ serotypes of HA showed that the $\mathrm{mAb}$ binds to a conserved quaternary epitope in the HA stem that comprises the F subdomain and the fusion peptide region of the neighbouring HA monomer. FI6 exhibited prophylactic efficacy in mice that were infected with $\mathrm{H} 1 \mathrm{~N} 1$ and $\mathrm{H} 3 \mathrm{~N} 2$ strains, and protected ferrets that were exposed to a highly pathogenic H5N1 strain.

Finally, Whittle et al. isolated plasma cells from a donor 1 week after vaccination with the 2007 trivalent vaccine, and isolated and sequenced rearranged heavy- and light-chain genes. They identified the $\mathrm{mAb} \mathrm{CH} 65$, which exhibited strikingly broad potency, neutralizing 30 out of $36 \mathrm{H} 1 \mathrm{~N} 1$ strains tested, including strains isolated as early as 1986 . Those strains that were resistant to CH65 exhibited a common mutation. The mAb bound to the HA globular head, mimicking the sialic acid interaction. It was thought that antibodies could not target this small area with such specificity.

The structural insight gained through these studies will potentially have a substantial impact on nextgeneration vaccine design.

\section{Sarah Crunkhorn}

ORIGINAL RESEARCH PAPERS Ekiert, D. C. et al. A highly conserved neutralizing epitope on group 2 influenza A viruses. Science 333, 843-850 (2011)| Corti, D. et al. A neutralizing antibody selected from plasma cells that binds to group 1 and group 2 influenza A hemagglutinins. Science $\mathbf{3 3 3}$, 850-856 (2011) | Whittle, J. R. R. et al. Broadly neutralizing human antibody that recognizes the receptor-binding pocket of influenza virus hemagglutinin. Proc. Natl Acad. Sci. USA 108, 14216-14221 (2011) 